\title{
Insulin receptor substrate-1 and Golgi phosphoprotein 3 are downstream targets of miR-126 in esophageal squamous cell carcinoma
}

\author{
HAOMIAO LI ${ }^{1 *}$, FANYU MENG $^{1 *}$, JUN MA $^{2}$, YONGKUI YU ${ }^{1}$, \\ XIONGHUAI HUA ${ }^{1}$, JIANJUN QIN ${ }^{1}$ and YIN LI $^{1}$ \\ ${ }^{1}$ Department of Thoracic Surgery, The Affiliated Tumor Hospital of Zhengzhou University, \\ Zhengzhou, Henan 450008; ${ }^{2}$ The Second Affiliated Hospital, Institute of Digestive Diseases, \\ Zhengzhou University, Zhengzhou, Henan 450014, P.R. China
}

Received March 21, 2014; Accepted June 24, 2014

DOI: $10.3892 /$ or.2014.3327

\begin{abstract}
Esophageal squamous cell carcinoma (ESCC) is a common histologic subtype in China. It has been suggested that abnormal expression of microRNAs (miRNAs) is associated with carcinogenesis. We investigated miR-126 expression and its potential targets in ESCC. The expression of miR-126 was detected in cancerous and paired paracancer tissues from 102 patients with ESCC. Target analysis of miR-126 was predicted using online tools. The effect of miR-126 expression on target proteins was assessed using miR-126 mimics or miR-126 inhibitors in ESCC cell lines. In addition, the impact of miR-126 on cell proliferation, apoptosis, migration and invasion was detected in ESCC cell lines. The expression of miR-126 was significantly lower in ESCC tissues, which was associated with tumor differentiation, lymph node metastasis, tumor in-depth and TNM stage. Insulin receptor substrate-1 (IRS-1) and Golgi phosphoprotein 3 (GOLPH3) were overexpressed in ESCC. Overexpression of IRS-1 was associated with cell differentiation, whereas GOLPH3 was related to lymph node metastasis, tumor invasion in-depth and TNM stage in ESCC patients. miR-126 mimics downregulated the expression of IRS-1 and GOLPH3 protein and suppressed the proliferation, migration and invasion of ESCC cells, whereas miR-126 inhibitors led to the opposite results. miR-126 suppressed the proliferation, migration and invasion of ESCC cells, and acted as a tumor suppressor in the carcinogenesis of ESCC. IRS-1 and GOLPH3 are downstream targets of miR-126 at the post-transcriptional level in ESCC.
\end{abstract}

Correspondence to: Dr Yin Li, Department of Thoracic Surgery, The Affiliated Tumor Hospital of Zhengzhou University, Zhengzhou, Henan 450008, P.R. China

E-mail: 654948226@qq.com

*Contributed equally

Key words: esophageal squamous cell carcinoma, miR-126, insulin receptor substrate-1, Golgi phosphoprotein 3, clinicopathological characteristics

\section{Introduction}

Esophageal cancer is the eighth most common cancer worldwide and the sixth most common cause of cancer-related mortality $(1,2)$. Although Barrett's adenocarcinoma is the most rapidly increasing cancer in Western countries, esophageal squamous cell carcinoma (ESCC) remains dominant in East Asia (3). Often diagnosed at later stages, the prognosis of affected patients is unsatisfactory despite the development of therapeutic options such as surgery, chemotherapy and radiotherapy (4). Consequently, there is a great need for ESCC biomarkers to allow a tailored multimodal approach with increased efficacy. Nevertheless, efforts to identify molecular markers in association with the pathogenesis of ESCC have, to date, been unsuccessful (5).

Mature microRNAs (miRNAs) are a class of small, wellconserved, non-coding RNA molecules that silence gene expression usually by interfering with mRNA stability or protein translation $(6,7)$. The function is performed by identifying 3'-UTRs (untranslated regions) of target mRNAs with conserved complementarities to the seed (nucleotides 2-7) of the miRNA. Up to $30 \%$ of human protein-coding genes may be regulated by miRNAs $(8,9)$. miRNAs are involved in biological and pathological processes including cell differentiation, proliferation, apoptosis and metabolism, and they are emerging as highly tissue-specific biomarkers with potential clinical application for defining cancer types and origins. miRNAs can either function as oncogenes or tumor suppressors $(10,11)$.

It has been reported that miR-126 is located on chromosome $9 \mathrm{q} 34.3$ within the host gene encoding for epidermal growth factor like-7 (EGFL-7), an endothelial cell-derived, secreted inhibitor of smooth muscle cell migration and a regulator of blood vessel formation $(12,13)$. Vasculature is required for the expansion of tumor masses, as inhibition of new vessel formation prevents tumor growth. Studies have shown that the expression of miR-126 is downregulated in cancers such as non-small cell lung cancer and colon cancer $(14,15)$, suggesting that the downregulation of miR-126 is significantly related to the occurrence and development of 
cancer. However, studies have also reported that aberrant overexpression of miR-126 contributes to carcinogenesis, suggesting that miR-126 acts as an oncogene in gastric cancer and oral squamous cell carcinoma $(16,17)$. Therefore, miR-126 may have diverse roles in tumor carcinogenesis. To date, some genes have been identified as miR-126 target genes, including sex determining region Y-box 2 (SOX2), Crk, vascular endothelial growth factor $(V E G F)$, matrix metalloproteinase-9 (MMP-9), insulin receptor substrate-1 (IRS-1), SLC7A5, TOM1, and CD97/G-protein-coupled receptor (GPCR) (16,18-21). Bioinformatics has also shown that the 3'-UTR of genes such as IRS-1, FBXO33, PTPN9, PLK2, and Golgi phosphoprotein 3 (GOLPH3) contains a putative binding site for miR-126.

However, the functional role and mechanism of miR-126 in regulating tumorigenesis of ESCC is still not fully understood. To identify miRNAs which can be specifically expressed and exert distinct biological actions in ESCC, we investigated miR-126 expression and its functional role in ESCC. miR-126 expression was measured in ESCC tissue specimens and paired paracancer tissues, and the relationship between the expression level of miR-126 and the clinicopathological characteristics were analyzed. Further functional studies of miR-126 showed that it could inhibit ESCC cell growth, migration and invasion. IRS-1 and GOLPH3 are potential downstream targets of miR-126 in ESCC.

\section{Materials and methods}

Tissue specimens and cancer cell lines. Specimens of 102 ESCC tissues and paired paracancer tissues were collected from Henan Cancer Hospital between July 2012 and July 2013. Clinicopathological data of patients were collected. None of the patients received preoperative antitumor therapy. All specimens were collected using two methods: one was snap frozen in liquid nitrogen immediately after surgery and stored at $-80^{\circ} \mathrm{C}$ for further miRNA detection, the other was formalin-fixed for histopathological assay. The clinicopathological information of the ESCC patients is shown in Table I. All patients provided informed consent under a protocol reviewed and approved by the Institutional Review Board, Henan Cancer Hospital.

The human ESCC cell line Eca9706 was purchased from the cell bank of the tumor hospital of the Chinese Academy of Medical Sciences/Biological Detection Center (Beijing, China). The cells were cultured in RPMI-1640 (Thermo, Waltham MA, USA) supplemented with $10 \%$ fetal bovine serum (FBS; Thermo) and penicillin/streptomycin in a $5 \%$ $\mathrm{CO}_{2}$ humidified incubator at $37^{\circ} \mathrm{C}$ (normal conditions for cell culture).

RNA isolation and real-time reverse transcription polymerase chain reaction ( $q R T-P C R$ ). Total-RNA in cancer tissues or cell lines was isolated using QIAzol lysis reagent and miRNeasy mini kit (Qiagen, Düsseldorf, Germany), according to the manufacturer's instructions. Total-RNA was isolated $48 \mathrm{~h}$ after cell transfection. Quantitation of total-RNA was carried out using spectrophotometry. All samples had an excellent 260/280 ratio. RNAs were reverse-transcripted into cDNAs using miScript Reverse Transcription kit (Qiagen) and stored at $-20^{\circ} \mathrm{C}$ for immediate or further use. The qRT-PCR of miRNA and mRNA expression was detected using miScript SYBR-Green PCR kit (Qiagen) on an ABI 7500 detector (Applied Biosystems). RUN6 was used as internal control according to the protocol. miR-126 miScript Primer assay (no. MS00003430), RNU6 miScript Primer assay (no. MS00033740), IRS-1 QuantiTect Primer assay (QT00074144), GOLPH3 QuantiTect Primer assay (no. QT00061320), and $\beta$-actin QuantiTect Primer assay (no. QT00095431) for miRNA or mRNA detection were commercially provided by Qiagen. Relative gene expression determinations were made using the comparative $\Delta \Delta \mathrm{Ct}$ method $\left(2^{-\Delta \Delta C t}\right)$. Patients were divided into two groups (high or low miR-126 expression) for further analysis. The high miR-126 expression group was defined as an expression level of miR-126 in tumor tissue higher than the paired paracancer tissue.

Online prediction of miRNA targets. To predict potential targets of miR-126, we searched PicTar (http://www.pictar.org), miRGenTargets (http://microrna.gr) and TargetScanHuman (http://www.targetscan.org/vert_61/). The 3'-UTR of IRS-1 mRNA (RefSeq 005544) and GOLPH3 mRNA (RefSeq 022130) may have putative miRNA-126 binding sites.

Immunohistochemistry. To explore expression of IRS-1 and GOLPH3 proteins in ESCC, immunohistochemistry experiments were carried out on the collected 3-5 $\mu \mathrm{m}$ tissue slices. After deparaffinization and antigen retrieval, slices were incubated at $4^{\circ} \mathrm{C}$ overnight with rabbit polyclonal GOLPH3 antibody (1:100; Abcam, Hong Kong), or rabbit polyclonal IRS-1 antibody (1:50; Santa Cruz Biotechnology, Inc., Santa Cruz, CA, USA; sc-559). After washing with phosphate-buffered saline (PBS) three times, the slides were incubated with biotinylated secondary antibody (1:80; goat anti-rabbit IgG, ready-to-use; Dako, Denmark) for $1 \mathrm{~h}$ at $37^{\circ} \mathrm{C}$, washing with PBS three times and chromogenic use of 3,3'-diaminobenzidine (DAB) for $5 \mathrm{~min}$. Counterstaining was carried out with hematoxylin. The immunohistochemically stained tissue sections were reviewed and scored separately by two pathologists blinded to the clinical information. Any intensity of cell membrane, endosome or cytoplasmic staining was considered a positive stain for GOLPH3, whereas cytoplasmic or nuclear staining was considered positive for IRS-1. Increased expression of proteins was considered if the percentage of stained cells was $\geq 10 \%$.

Transfection of miR-126 mimics or inhibitor into ESCC cells. Eca9706 cells were seeded into 24-well plates and transfected when the cells reached $60 \%$ confluence. Syn-hsa-miR-126-3p miScript miRNA Mimic (no. MSY0000445), anti-hsamiR-126-3p miScript miRNA Inhibitor (no. MIN0000445), and miScript miRNA Inhibitor Negative Control (mock sequence, no. 1027271), commercially provided by Qiagen, were transfected into Eca9706 cells. siRNAs were allowed to form transfection complexes with HiPerFect Transfection Reagent (Qiagen) in serum-free Opti-MEM, according to the manufacturer's protocol. All groups were performed in triplicate. Cells were collected for RNA or protein assay at different time points after transfection.

For the invasion and apoptosis assays, cells were seeded in 6-well plates and the transfected cells were suspended using tyrosine; a hemocytometer was used for cell counting. 
Table I. Expression of miR-126, IRS-1 protein and GOLPH3 protein in ESCC patients.

\begin{tabular}{|c|c|c|c|c|c|c|c|}
\hline Variable & Total & miR-126 high & P-value & IRS-1-positive & P-value & GOLPH3-positive & P-value \\
\hline Age (years) & & & 0.298 & & 0.68 & & 0.833 \\
\hline$<60$ & 45 & 13 & & 32 & & 14 & \\
\hline$\geq 60$ & 57 & 23 & & 34 & & 20 & \\
\hline Gender & & & 0.513 & & 0.275 & & 0.408 \\
\hline Male & 69 & 26 & & 43 & & 22 & \\
\hline Female & 33 & 10 & & 23 & & 12 & \\
\hline Tumor location & & & 0.413 & & 0.788 & & 0.582 \\
\hline Upper & 15 & 4 & & 11 & & 4 & \\
\hline Middle & 68 & 23 & & 45 & & 25 & \\
\hline Lower & 19 & 9 & & 10 & & 5 & \\
\hline Differentiation & & & 0.017 & & 0.006 & & 0.462 \\
\hline Well & 29 & 10 & & 19 & & 7 & \\
\hline Moderate & 24 & 14 & & 10 & & 9 & \\
\hline Poor & 49 & 12 & & 37 & & 18 & \\
\hline Smoking & & & 0.539 & & 0.539 & & 0.294 \\
\hline Yes & 44 & 14 & & 30 & & 12 & \\
\hline No & 58 & 22 & & 36 & & 22 & \\
\hline Alcoholic & & & 0.477 & & 0.477 & & 0.814 \\
\hline Yes & 26 & 11 & & 15 & & 8 & \\
\hline No & 76 & 25 & & 51 & & 26 & \\
\hline Lymph node metastasis & & & 0.019 & & 0.289 & & 0.01 \\
\hline Yes & 39 & 8 & & 31 & & 7 & \\
\hline No & 63 & 28 & & 35 & & 27 & \\
\hline Invasion depth & & & 0.009 & & 0.515 & & $<0.01$ \\
\hline $\mathrm{T} 1$ & 12 & 9 & & 3 & & 11 & \\
\hline $\mathrm{T} 2$ & 34 & 11 & & 23 & & 12 & \\
\hline $\mathrm{T} 3,4$ & 56 & 16 & & 40 & & 11 & \\
\hline TNM stage & & & 0.03 & & 0.406 & & $<0.01$ \\
\hline I & 10 & 7 & & 3 & & 9 & \\
\hline II & 59 & 21 & & 38 & & 18 & \\
\hline III & 33 & 8 & & 25 & & 7 & \\
\hline
\end{tabular}

Relationship between expression levels of miR-126, IRS-1 protein, GOLPH3 protein and clinicopathological characteristics in 102 patients with ESCC analyzed using the Fisher's exact test. miR-126 high is defined as an expression level of miR-126 in tumor tissue higher than the paired paracancerous tissue. IRS-1- or GOLPH3-positive are defined as the percentage of immunoreactive cells $\geq 10 \%$.

Western blot analysis. Transfected cells were lysed in RIPA buffer (Sigma, St. Louis, MO, USA) with protease inhibitors. Proteins were resolved in 10\% SDS-PAGE gel and electro transferred onto a PVDF membrane, blocked with 5\% milk, and then the membrane was incubated with a primary antibody at $4^{\circ} \mathrm{C}$ overnight (rabbit polyclonal GOLPH3 antibody, 1:500; Abcam, or rabbit polyclonal IRS-1 antibody; 1:500; Santa Cruz). After washing with Tris-buffered saline 3 times, corresponding horseradish peroxidase-conjugated secondary antibody $(1: 2,000$; goat anti-rabbit IgG; Dako) was used to incubate the membrane at room temperature for $2 \mathrm{~h}$. The membrane was then washed 3 times and the blots were visualized using Western Lightning Ultra (PerkinElmer, Waltham, MA, USA). Bands were quantified using FluorChem FC3
Software ${ }^{\circledR}$ (ProteinSimple, San Francisco, CA, USA) for band intensity and $\beta$-actin was used as internal control.

Cell proliferation assay. The cells transfected with $50 \mathrm{nM}$ miR-126 mimics, inhibitors or mock sequence were initially seeded at a density of $5.0 \times 10^{3}$ cells/well in 96 -well plates with siRNAs. After 6-8 h, non-adherent cells were removed by washing with PBS. The remaining adherent cells were cultured under normal conditions, and this time point was set as the start point. Cell counting kit-8 (CCK-8) analyses were performed at 5, 24, 48, 72 or $96 \mathrm{~h}$ for cell proliferation observation. Absorbance was detected at $490 \mathrm{~nm}$ in an enzyme microplate reader (Wellscan MK-3; Labsystems Dragon, Finland). 
A

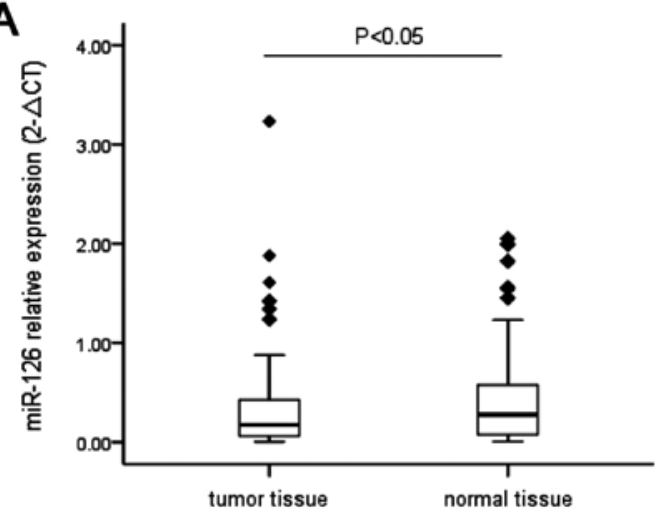

B

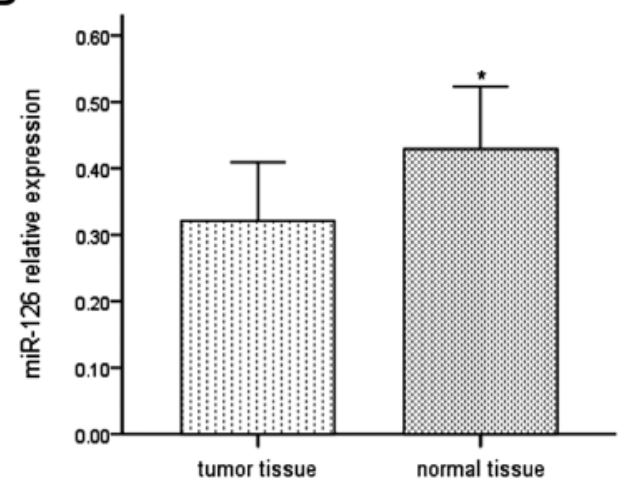

Figure 1. Downregulation of miR-126 in ESCC tissue specimens. miR-126 expression was analyzed by real-time reverse transcriptase polymerase chain reaction and normalized to U6 expression. (A) Lower expression of miR-126 in ESCC tissues compared with normal tissue in 102 ESCC patients. (B) The mean expression level of miR-126 in 102 ESCC tissues and paired paracancer tissues. ${ }^{*} \mathrm{P}<0.05$.

Cell apoptosis assay. Cells were suspended in $0.25 \%$ trypsin without EDTA and harvested at $48 \mathrm{~h}$ after transfection. After washing twice with PBS, the cell concentration was adjusted to $1 \times 10^{6}$ using Annexin V-FITC binding buffer. Then, $100 \mu 1$ of the suspended cells were stained with $5 \mu \mathrm{l}$ of Annexin $\mathrm{V}$ for $30 \mathrm{~min}$ and $10 \mu \mathrm{l}$ of propidium iodide (4Abio, Beijing, China) for $15 \mathrm{~min}$ in the dark at room temperature. After washing with PBS, $400 \mu 1$ of PBS was added to the samples and analysis was performed using a flow cytometer (BD Biosciences, San Jose, CA, USA). The percentage of Annexin V-positive cells was recorded for analysis.

Cell migration and invasion assay. The Transwell invasion system $(8.0 \mu \mathrm{m}$ polycarbonate membrane, $6.5 \mathrm{~mm}$ insert in a 6-well plate; Corning, Lowell, MA, USA) with or without Matrigel was used for the invasion or migration assay. After transfection for $24 \mathrm{~h}$, cells were harvested using $0.25 \%$ trypsin-EDTA (Thermo). For the migration assay, $2 \times 10^{6}$ cells were added into the upper compartment of the Transwell chamber. For the invasion assay, $1 \times 10^{5}$ cells were plated on chambers preloaded with Matrigel. In both assays, all cells were suspended in $100 \mu \mathrm{l}$ of RPMI-1640 without serum, 1,000 $\mu$ l RPMI-1640 supplemented with 10\% FBS was added in the bottom compartment of the chamber. After $12 \mathrm{~h}$ of incubation, Matrigel, dead cells and non-migrated or non-invaded cells on the upper surface of the membrane were removed with a cotton swab. The membranes were fixed with $4 \%$ paraformaldehyde and stained with $1 \%$ crystal purple. The invasive cells that were stuck to the lower surface of the membrane were then counted.

Statistical analysis. Statistical analysis was performed using SPSS 17.0 software (SPSS, Inc., Chicago, IL, USA). Data obtained from qRT-PCR were $\log _{2}$ transformed. Differences in miR-126 expressions between ESCC and paired paracancer tissues were analyzed using non-parametric test. The two-sided Fisher's exact test was used to determine the relationship between miR-126, IRS-1 and GOLPH3 expression and clinicopathological variables. Quantitative data expressed as means \pm standard deviation and differences between groups were calculated with the t-test. All results were considered statistically significant when $\mathrm{P}<0.05$.

\section{Results}

Downregulation of miR-126 expression and association with lymph node metastasis, tumor in-depth or cell differentiation in ESCC. To investigate the expression of miR-126 in ESCC, we first evaluated the status of miR-126 in samples of ESCC by RT-qPCR. Total-RNA was isolated from 102 pairs of ESCC and paracancer tissues. Lower expression of miR-126 was found in $66(64.7 \%)$ patients. The expression of miR-126 in ESCC samples was on average decreased $\sim 68.36 \%$ of that in paracancer samples $(\mathrm{P}<0.05$; Fig. $1 \mathrm{~A}$ and $\mathrm{B})$.

To further investigate the relationship between the expression level of miR-126 and clinicopathological characteristics, all patients were divided into two groups. Lymph node metastasis, tumor in-depth, cell differentiation and TNM stage were all associated with miR-126 expression. Greater possibility of lymph node metastasis was observed in the miR-126 low expression group. There were no statistically significant associations between miR-126 expression and other clinicopathological parameters, such as gender, age, tumor location, alcoholism or smoking ( $\mathrm{P}>0.05$; Table I).

\section{IRS-1 and GOLPH3 are potential targets of miR-126 predicted} online. Since miR-126 was closely related to clinicopathological characteristics in ESCC, we further analyzed its function and molecular mechanism in the carcinogenesis of ESCC. We predicted targets of miR-126 in humans using the online tools miRGenTargets, TargetScanHuman and PicTar. Following computational prediction, the proteins IRS-1, FBXO33, PLK2, PTPN9, and GOLPH3 were analyzed. We reviewed published reports that focused on individual miRNAs of the proteins. Among them, IRS-1 has been reported as a target of miR-126 in colon cancer, whereas GOLPH3 shows overexpression in ESCC and is associated with poor prognosis. Therefore IRS-1 and GOLPH3 were selected as targets of our research (Fig. 2A).

IRS-1 and GOLPH3 are overexpressed in human ESCC. To verify our prediction, we first studied the relationship between the two proteins in ESCC. IRS-1 and GOLPH3 were determined using immunohistochemistry in ESCC tissues and the paracancer tissues respectively. The expression of IRS-1 


\section{A}

\author{
Position 131-137 of IRS1 3' UTR \\ hsa-miR-126
}

Position 179-185 of GOLPH3 3' UTR

hsa-miR-126

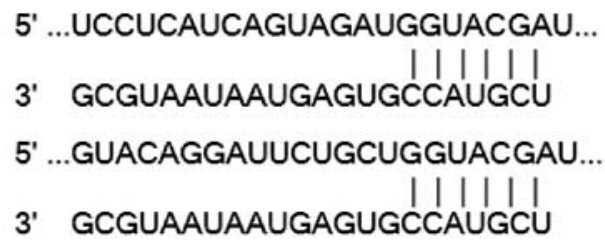

B
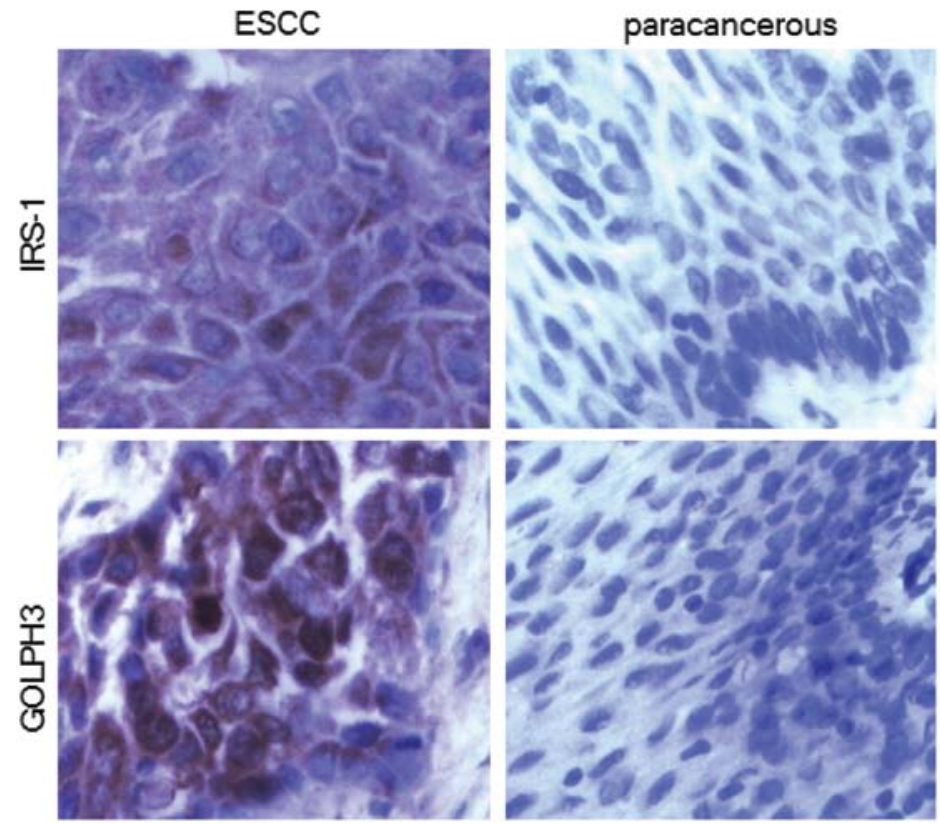

Figure 2. IRS-1 and GOLPH3 are potential targets of miR-126 and are overexpressed in human ESCC tissues. (A) Position of the miR-126 target site in the 3'-UTR of the IRS-1 or GOLPH3 mRNA predicted by TargetScan or PigTar, a putative miR-126 binding site that encompasses 6 perfectly matched nucleotides was defined at the 3'-UTR of IRS-1 or GOLPH3. (B) Immunohistochemical analysis of proteins in tumor or paired paracancer tissues.

protein was significantly higher in ESCC samples compared with paracancer tissues, and the same results were found for GOLPH3 expression levels ( $\mathrm{P}<0.01$; Fig. 2B).

We also analyzed the relationship between IRS-1 or GOLPH3 protein and clinical characteristics. Positive expression of IRS-1 was associated with cell differentiation but no obvious association with any other clinical characteristics. Meanwhile, GOLPH3 was closely related to lymph node metastasis, tumor invasion in-depth and TNM stage (Table I).

IRS-1 and GOLPH3 protein expression after up/down regulation of $m i R-126$. To further explore the impact of aberrant expression of miR-126 on IRS-1 and GOLPH3, we transfected miR-126 mimics, inhibitors or mock sequence into Eca9706 cells. miR-126 levels increased on an average 5.17-fold $(\mathrm{P}<0.01)$ after transfection with miR-126 mimics and decreased 0.37 -fold $(\mathrm{P}<0.01)$ when transfected with miR-126 inhibitors. There was no obvious change in cells transfected with the mock sequence (Fig. 3A).

Initially, relative mRNA levels of IRS-1 and GOLPH3 in Eca9706 cells were detected. Then, $50 \mathrm{nM}$ of miR-126 mimics, inhibitors or mock sequence were transfected into Eca9706 cells. After transfection for $48 \mathrm{~h}$, we detected the mRNA levels of IRS-1 and GOLPH3 in these cells. In cells transfected with miR-126 mimics, altered relative mRNA levels of IRS-1 (1.05-fold) and GOLPH3 (0.94-fold) had no statistical differ- ence compared with non-treated controls. Downregulation of miR-126 in Eca9706 cells showed no significant difference in IRS-1 (0.92-fold) and GOLPH3 (0.92-fold) mRNA levels, and there were also no differences in the mock sequence group ( $\mathrm{P}>0.05$; Fig. 3B).

We then analyzed the relationship between miR-126 and IRS-1 or GOLPH3 protein. The expression level of IRS-1 protein decreased 0.71-fold $(\mathrm{P}<0.05)$ compared with the non-treated control, and GOLPH3 protein decreased 0.66-fold $(\mathrm{P}<0.01)$ when transfected with miR-126 mimics. In addition, when transfected with miR-126 inhibitors, IRS-1 protein expression increased 2.01-fold $(\mathrm{P}<0.01)$ and GOLPH3 protein increased 2.14-fold $(\mathrm{P}<0.01)$ compared with the non-treated controls, and there were no differences between the mock sequence group and the non-treated controls (Fig. 3C and D).

miR-126 suppresses cell proliferation but has no effect on apoptosis in ESCC cells. To further characterize the functional role of miR-126 in ESCC, we observed the effect of miR-126 on the proliferation and apoptosis of ESCC cells. After transfection with miR-126 mimics, cell proliferation was suppressed after $48 \mathrm{~h}(0.433 \pm 0.054$ vs. $0.514 \pm 0.061, \mathrm{P}<0.05)$ and $72 \mathrm{~h}(0.680 \pm 0.038$ vs. $0.943 \pm 0.070, \mathrm{P}<0.01)$. In addition, transfection with miR-126 inhibitors showed an improvement in cell proliferation at $48 \mathrm{~h}(0.584 \pm 0.088$ vs. $0.514 \pm 0.061$, $\mathrm{P}<0.05)$ and $72 \mathrm{~h}(1.130 \pm 0.098$ vs. $0.943 \pm 0.070, \mathrm{P}<0.01)$; no 
A

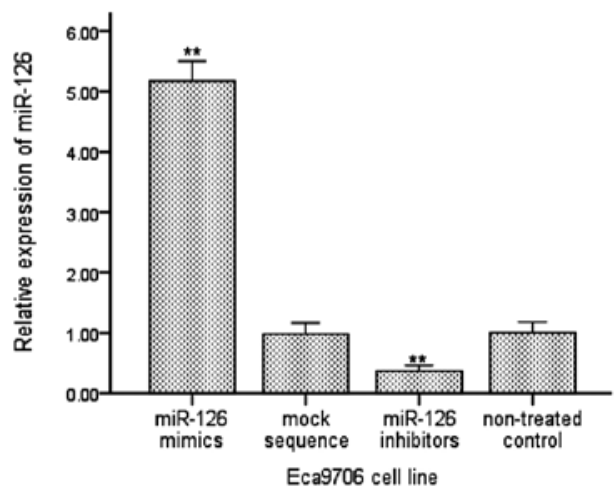

C

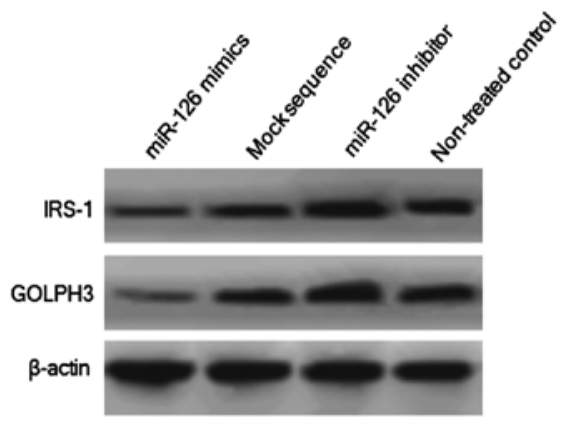

B

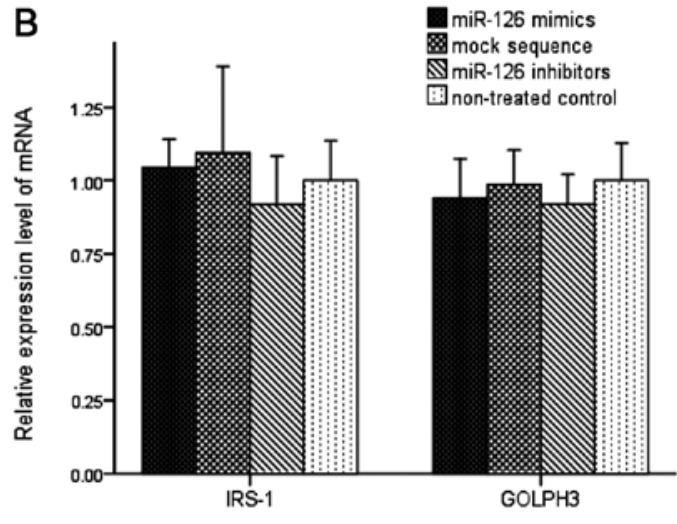

D

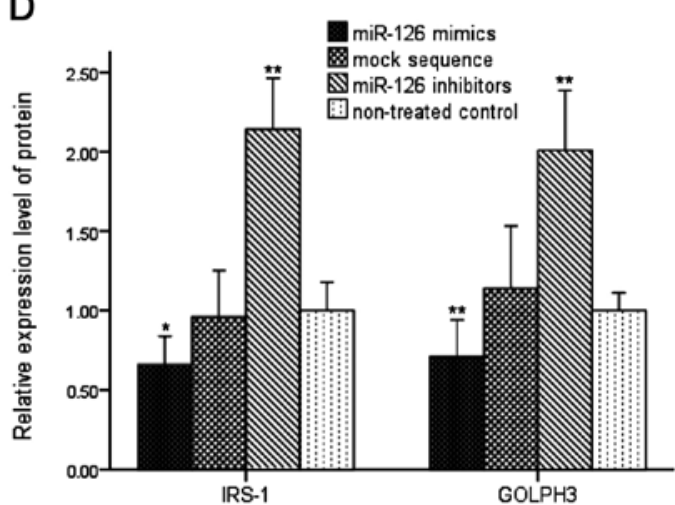

Figure 3. Effects of miR-126 on mRNA or protein expression of IRS-1 and GOLPH3. (A) Levels of miR-126 in Eca9706 cells were detected $24 \mathrm{~h}$ after transfection, the miR-126 level increased after transfection with miR-126 mimics and decreased when transfected with miR-126 inhibitors; there was no obvious change in cells transfected with mock sequence compared with non-treated controls. (B) Relative expression level of IRS-1 and GOLPH3 mRNA in Eca9706 cells after transfection with $50 \mathrm{nM}$ of miR-126 mimics, miR-126 inhibitors or mock sequence; there was no significant change in mRNA levels in all groups compared with non-treated controls. (C and D) Relative expression of IRS-1 and GOLPH3 protein were detected using western blotting. IRS-1 and GOLPH3 protein levels showed a significant increase after downregulation of miR-126, whereas the expression of the 2 proteins decreased with upregulated miR-126 in Eca9706 cells. ${ }^{*} \mathrm{P}<0.05,{ }^{* *} \mathrm{P}<0.01$.

alteration of cell proliferation occurred in the mock sequence group ( $\mathrm{P}>0.05$; Fig. 4A) compared with the non-treated group. However, cell apoptosis, which was measured using flow cytometry at $48 \mathrm{~h}$ after transfection, showed no significant differences between all three groups compared to that in the non-treated controls ( $\mathrm{P}>0.05$, Fig. 4B and $\mathrm{C})$.

miR-126 regulates cell migration and invasion. To explore the correlation between miR-126 expression and migration or invasion potential in human ESCC cell lines, we carried out the Transwell assay. To reduce the bias of proliferation suppression of miR-126, the incubation time was controlled for $24 \mathrm{~h}$. In the migration assay, the number of migrated cells was significantly decreased in the group transfected with miR-126 mimics compared to the non-treated controls $(43.87 \pm 7.59$ vs. $67.77 \pm 10.57, \mathrm{P}<0.01)$, whereas the number of cells increased in the miR-126 inhibitor group $(89.59 \pm 12.16$ vs. $67.77 \pm 10.57$, $\mathrm{P}<0.01$, Fig. 5A and B). In the invasion assay, cell invasiveness increased significantly when transfected with the miR-126 inhibitor $(48.21 \pm 10.21$ vs. $27.03 \pm 8.36, \mathrm{P}<0.01)$, whereas overexpression of miR-126 significantly suppressed Eca9706 cell invasion $(14.44 \pm 6.14$ vs. $27.03 \pm 8.36, \mathrm{P}<0.01)$; results in the mock sequence group were similar to the non-treated controls (P>0.05, Fig. 5A).

\section{Discussion}

miRNAs have important roles in tumorigenesis by various mechanisms. miRNAs can act as tumor suppressors depending on the targeting of specific suppressor genes or oncogenes, or they can alter tumorous epithelial-mesenchymal transitions to promote tumor invasion and metastasis, and they can also regulate the expression of metastasis-associated genes in tumors $(22,23)$. Thus, the aberrant expression of tumorsuppressive miRNAs may contribute to human carcinogenesis. For example, downregulation of miR-7 can promote metastasis of gastric cancer by targeting the insulin-like growth factor-1 receptor, and the low level of miR-210 is associated with the carcinogenesis of ESCC $(24,25)$. The expression of miR-126 is downregulated in many cancers, such as lung, breast and liver cancer $(14,26)$, suggesting that the suppression of miR-126 was significantly related to the occurrence and development of cancer. The results of the present study confirmed that miRNA-126 was downregulated in ESCC compared with paracancer tissues, indicating that miR-126 may be associated with the carcinogenesis of ESCC. We therefore analyzed the relationship between downregulation of miR-126 and the clinicopathological characteristics of ESCC patients. We found that downregulation of miR-126 was associated 

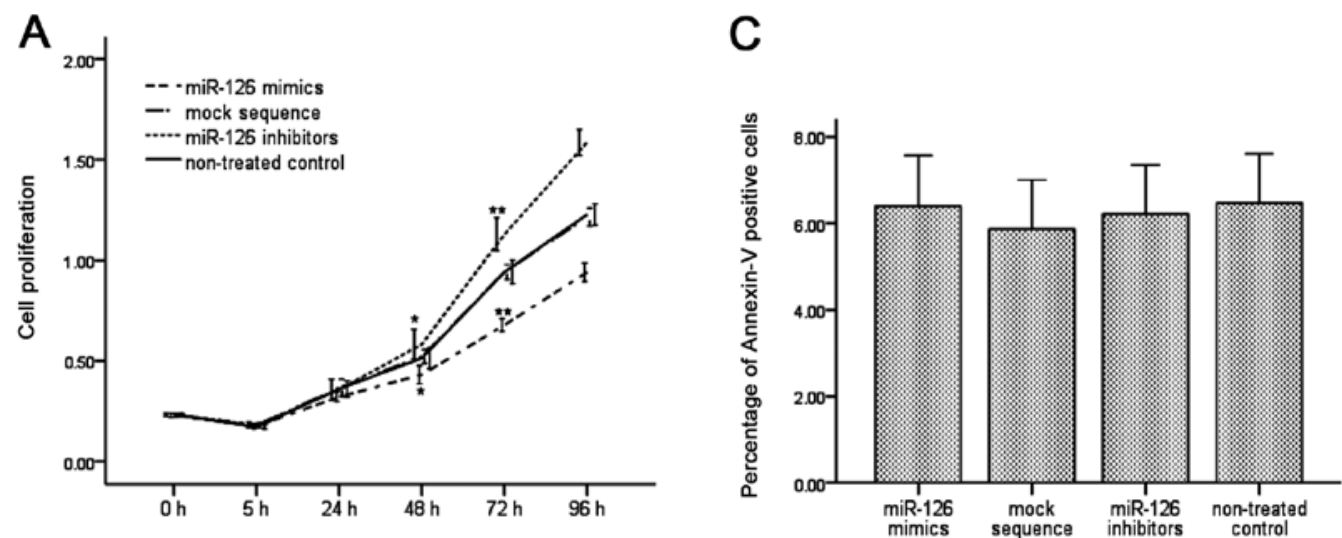

B
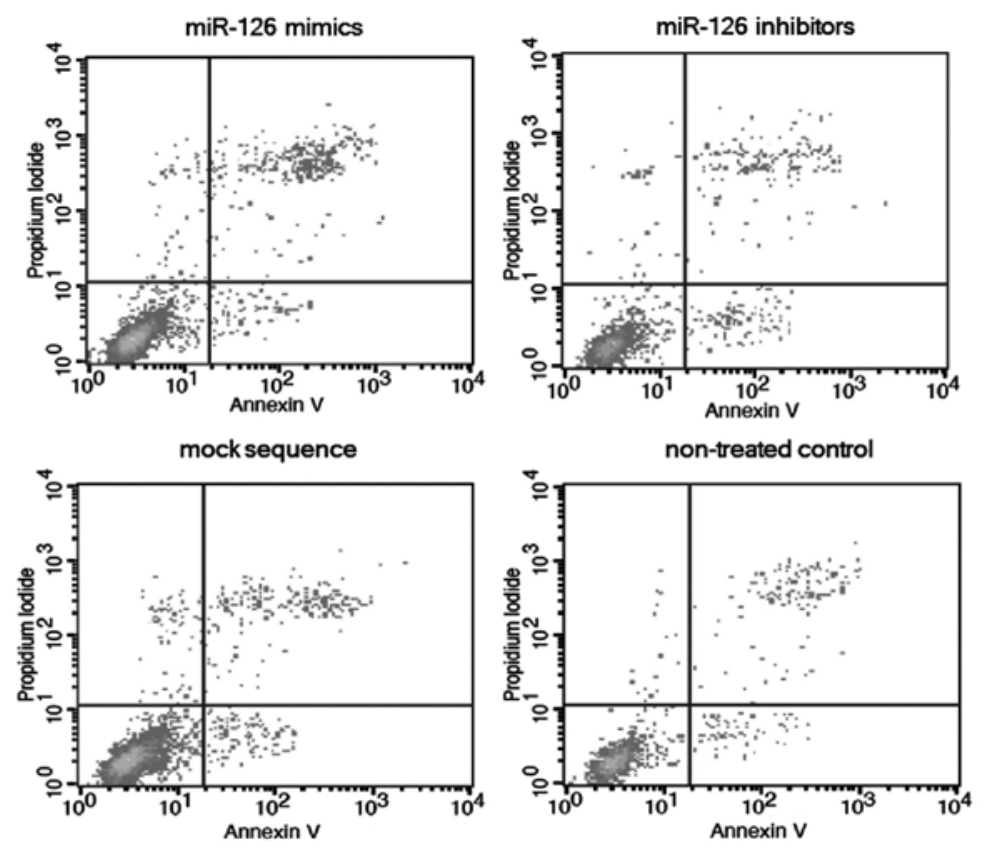

Figure 4. Effect of miR-126 on cell proliferation and apoptosis in the Eca9706 cell line. (A) Cell proliferation significantly increased 48 and $72 \mathrm{~h}$ after transfecting miR-126 inhibitors, whereas upregulation of miR-126 led to a decrease of cell proliferation. (B and C) miR-126 on cell apoptosis. Neither the miR-126 mimics nor the inhibitors caused a statistical change in cell apoptosis. ${ }^{*} \mathrm{P}<0.05,{ }^{* *} \mathrm{P}<0.01$.
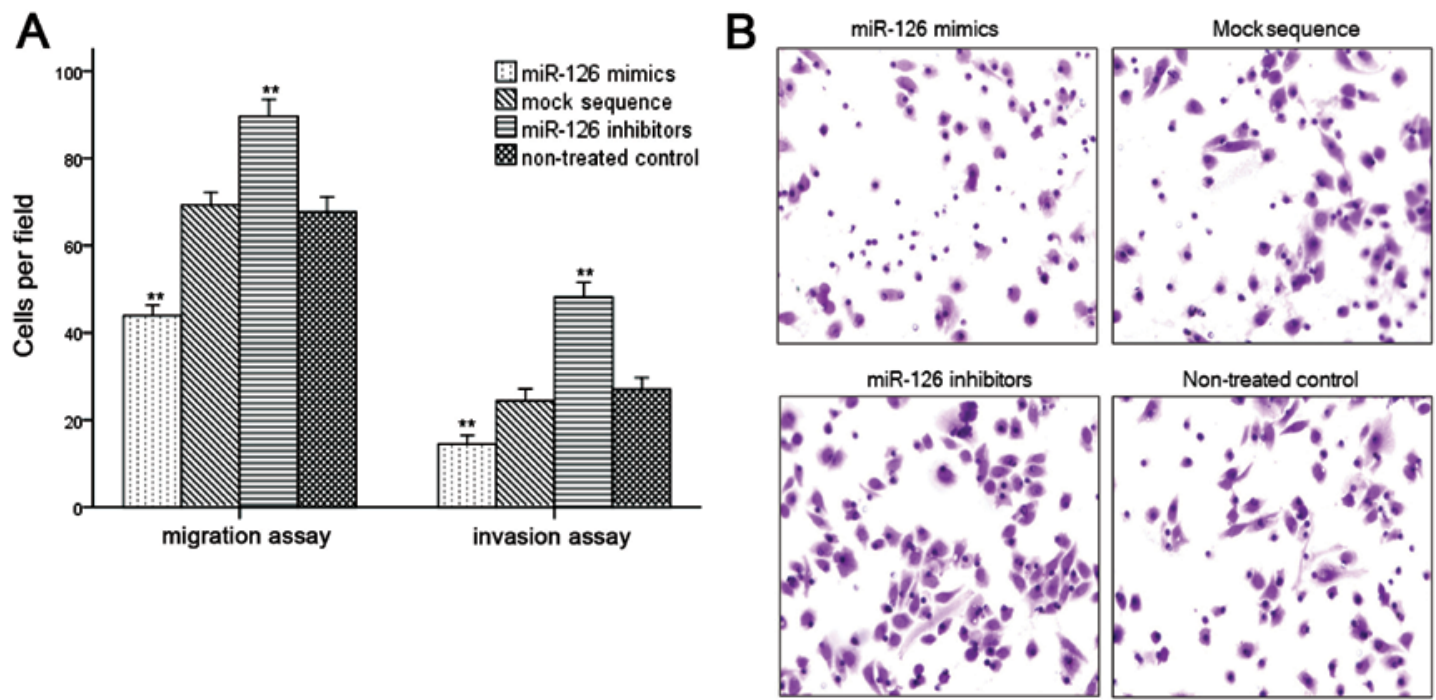

Figure 5. Effect of miR-126 on cell invasion and migration in vitro. (A) Results of cell invasion and migration assay. After incubation for $12 \mathrm{~h}$, the invaded or migrated cells that penetrated to the lower surface of the membrane were counted from 5 randomly selected visual fields. (B) In the migration assay, downregulation of miR-126 promoted cell migration, whereas upregulation of miR-126 caused a decrease of migrated cells. ${ }^{* *} \mathrm{P}<0.01$. 
with tumor cell differentiation, lymph nodes metastasis and tumor invasion in-depth in ESCC patients, which indicated that miR-126 acts as an oncogene in ESCC and participates in tumor invasiveness and metastasis. This result is similar to that found in colon and lung cancer $(18,20)$.

To further explore the molecular mechanism of miR-126 in suppressing ESCC development and invasion, we predicted IRS-1 and GOLPH3 as novel targets of miR-126. The insulin receptor substrates are cytoplasmic signaling adaptor proteins that function as intermediates of the insulin receptor and insulin-like growth factor receptor (IGF-IR), which are involved in cell growth and survival. As a member of the IRS family, IRS-1 has been a hotspot and is widely expressed. Tyrosine-phosphorylated IRS-1 bind proteins containing Src homology 2 (SH2) domains, which activate mitogen-activated protein kinase (MAPK) by effector cascades such as the PI3K pathway (27). Porter et al (28) reported that IRS-1 was highly expressed in localized breast tumors and that high IRS-1 levels can be a good indicator of the effectiveness of specific types of chemotherapy in breast cancer, but were not related to tumor aggressiveness. Several studies have also shown that IRS-1 forced expression of VEGF expression via transcriptional activation and promoted cell proliferation in human colon cancer (29,30). The results of the present study showed that IRS-1 was overexpressed in ESCC tissues and was associated with cell differentiation. Forced expression of miR-126 downregulated the expression level of IRS-1 protein in Eca9706 cells. Therefore, we suggest that IRS-1 is a direct target of miR-126 in human ESCC.

The endocytic protein, GOLPH3, is a highly conserved $34 \mathrm{kDa}$ protein which was initially identified by proteomic characterization of the Golgi apparatus. It was suggested an enhanced activation of mTOR signaling represents a molecular basis of the oncogenic activity of GOLPH3 (31). Several studies have reported that the increased expression of GOLPH3 is closely associated with the clinicopathological characteristics of cancers, such as prostate, lung, ovary and gastric cancer (31-33). Wang et al (34) showed that GOLPH3 was overexpressed in ESCC and was positively associated with clinical stage, TNM classification, histological differentiation and vital status. The present study indicates that GOLPH3 is highly expressed in ESCC and is related to tumor invasion in-depth, lymph nodes metastasis and TNM stage, which is the same as the above study. However, in our study, expression of GOLPH3 was not related to histological differentiation. Although there are few differences, results from the present study and previous studies suggest that GOLPH3, as well as IRS-1, are targets of miR-126 in ESCC, indicating that miR-126 can have different targets and roles across malignancies. In the present study, the mRNA levels of IRS-1 and GOLPH3 did not change significantly with miR-126 levels (when up/downregulated) in ESCC cell lines. This indicated that IRS-1 and GOLPH3 were negatively regulated by miR-126 at the post-transcriptional level in ESCC.

Crawford et al (18) indicated that miR-126 inhibited the proliferation of the human lung cancer cell line A549 both in vitro and in vivo. Li et al (35) found that miR-126 suppresses the proliferation and invasion of the human colon cancer cell lines HCT116, SW620 and HT-29. However, the functions of miR-126 in esophageal cancer progression remain unresolved.
In the present study, the overexpression of miR-126 suppressed cell proliferation in the ESCC cell line Eca9706. Moreover, the Transwell experiment also showed that miR-126 can inhibit ESCC cell migration and invasion in vitro. Collectively, these results suggest that miR-126 may function as a tumor suppressor by inhibiting cell proliferation, suppressing migration and invasion, which, in turn, affect multiple clinicopathological characteristics of cancer such as lymph node metastasis and depth of invasion. At the same time, this is the first report indicating the suppressor action of miR-126 in ESCC.

In conclusion, as a tumor suppressor, miR-126 expression decreased in ESCC and might have a tumor suppression role via the regulation of IRS-1 and GOLPH3. It can suppress cell proliferation, migration and invasion. miR-126 correlates with lymph node metastasis, tumor invasion in-depth and TNM stage of ESCC, and could serve as a potential marker and therapeutic target in ESCC.

\section{Acknowledgements}

This study was supported by the Research Fund for the National Key Clinical Speciality of China, and the Innovation Foundation of Excellent Intellectuals of Henan Province (no. 20070214).

\section{References}

1. Ferlay J, Shin HR, Bray F, Forman D, Mathers C and Parkin DM: Estimates of worldwide burden of cancer in 2008: GLOBOCAN 2008. Int J Cancer 127: 2893-2917, 2010.

2. Jemal A, Siegel R, Xu J and Ward E: Cancer statistics, 2010. CA Cancer J Clin 60: 277-300, 2010.

3. Mathé EA, Nguyen GH, Bowman ED, et al: MicroRNA expression in squamous cell carcinoma and adenocarcinoma of the esophagus: associations with survival. Clin Cancer Res 15: 6192-6200, 2009.

4. Song QK, Li J, Jiang HD, He YM, Zhou XQ and Huang CY: Esophageal cancer mortality during 2004-2009 in Yanting County, China. Asian Pac J Cancer Prev 13: 5003-5006, 2012.

5. Fareed KR, Kaye P, Soomro IN, et al: Biomarkers of response to therapy in oesophago-gastric cancer. Gut 58: 127-143, 2009.

6. Kloosterman WP and Plasterk RH: The diverse functions of microRNAs in animal development and disease. Dev Cell 11: 441-450, 2006.

7. Pillai RS: MicroRNA function: multiple mechanisms for a tiny RNA? RNA 11: 1753-1761, 2005.

8. Lewis BP, Burge CB and Bartel DP: Conserved seed pairing, often flanked by adenosines, indicates that thousands of human genes are microRNA targets. Cell 120: 15-20, 2005.

9. Croce CM and Calin GA: miRNAs, cancer, and stem cell division. Cell 122: 6-7, 2005.

10. Rosenfeld N, Aharonov R, Meiri E, et al: MicroRNAs accurately identify cancer tissue origin. Nat Biotechnol 26: 462-469, 2008.

11. Croce CM: Causes and consequences of microRNA dysregulation in cancer. Nat Rev Genet 10: 704-714, 2009.

12. Schmidt M, De Maziere A, Smyczek T, et al: The role of Egfl7 in vascular morphogenesis. Novartis Found Symp 283: 18-28; discussion 28-36, 238-241, 2007.

13. Schmidt M, Paes K, De Maziere A, et al: EGFL7 regulates the collective migration of endothelial cells by restricting their spatial distribution. Development 134: 2913-2923, 2007.

14. Li XM, Wang AM, Zhang J and Yi H: Downregulation of miR-126 expression in colorectal cancer and its clinical significance. Med Oncol 28: 1054-1057, 2011.

15. Yang J, Lan H, Huang X, Liu B and Tong Y: MicroRNA-126 inhibits tumor cell growth and its expression level correlates with poor survival in non-small cell lung cancer patients. PLoS One 7: e42978, 2012.

16. Otsubo T, Akiyama Y, Hashimoto Y, Shimada S, Goto K and Yuasa Y: MicroRNA-126 inhibits SOX2 expression and contributes to gastric carcinogenesis. PLoS One 6: e16617, 2011. 
17. Sasahira T, Kurihara M, Bhawal UK, et al: Downregulation of miR-126 induces angiogenesis and lymphangiogenesis by activation of VEGF-A in oral cancer. Br J Cancer 107: 700-706, 2012.

18. Crawford M, Brawner E, Batte K, et al: MicroRNA-126 inhibits invasion in non-small cell lung carcinoma cell lines. Biochem Biophys Res Commun 373: 607-612, 2008.

19. Ye P, Liu J, He F, Xu W and Yao K: Hypoxia-induced deregulation of miR-126 and its regulative effect on VEGF and MMP-9 expression. Int J Med Sci 11: 17-23, 2014.

20. Li N, Li X, Huang S, Shen S and Wang X: miR-126 inhibits colon cancer proliferation and invasion through targeting IRS1, SLC7A5 and TOM1 gene. Zhong Nan Da Xue Xue Bao Yi Xue Ban 38: 809-817, 2013 (In Chinese).

21. Lu YY, Sweredoski MJ, Huss D, Lansford R, Hess S and Tirrell DA: Prometastatic GPCR CD97 is a direct target of tumor suppressor microRNA-126. ACS Chem Biol 9: 334-338, 2014.

22. Kalluri R and Weinberg RA: The basics of epithelial-mesenchymal transition. J Clin Invest 119: 1420-1428, 2009.

23. Zhang Y, Wang Z, Chen M, et al: MicroRNA-143 targets MACC 1 to inhibit cell invasion and migration in colorectal cancer. Mol Cancer 11: 23, 2012

24. Zhao X, Dou W, He L, et al: MicroRNA-7 functions as an antimetastatic microRNA in gastric cancer by targeting insulin-like growth factor-1 receptor. Oncogene 32: 1363-1372, 2013.

25. Tsuchiya S: The role of microRNA-210 in esophageal squamous cell carcinoma. Yakugaku Zasshi 132: 1069-1073, 2012 (In Japanese).

26. Yang R, Dick M, Marme F, et al: Genetic variants within miR-126 and miR-335 are not associated with breast cancer risk. Breast Cancer Res Treat 127: 549-554, 2011.
27. White MF: IRS proteins and the common path to diabetes. Am J Physiol Endocrinol Metab 283: E413-E422, 2002.

28. Porter HA, Perry A, Kingsley C, Tran NL and Keegan AD: IRS1 is highly expressed in localized breast tumors and regulates the sensitivity of breast cancer cells to chemotherapy, while IRS2 is highly expressed in invasive breast tumors. Cancer Lett 338: 239-248, 2013

29. Shi B, Sepp-Lorenzino L, Prisco M, Linsley P, deAngelis T and Baserga R: Micro RNA 145 targets the insulin receptor substrate-1 and inhibits the growth of colon cancer cells. J Biol Chem 282: 32582-32590, 2007

30. Yin Y, Yan ZP, Lu NN, et al: Downregulation of miR-145 associated with cancer progression and VEGF transcriptional activation by targeting N-RAS and IRS1. Biochim Biophys Acta 1829: 239-247, 2013

31. Scott KL, Kabbarah O, Liang MC, et al: GOLPH3 modulates mTOR signalling and rapamycin sensitivity in cancer. Nature 459: 1085-1090, 2009

32. Hua X, Yu L, Pan W, et al: Increased expression of Golgi phosphoprotein-3 is associated with tumor aggressiveness and poor prognosis of prostate cancer. Diagn Pathol 7: 127, 2012.

33. Hu BS, Hu H, Zhu CY, Gu YL and Li JP: Overexpression of GOLPH3 is associated with poor clinical outcome in gastric cancer. Tumour Biol 34: 515-520, 2013.

34. Wang JH, Chen XT, Wen ZS, et al: High expression of GOLPH3 in esophageal squamous cell carcinoma correlates with poor prognosis. PLoS One 7: e45622, 2012.

35. Li N, Tang A, Huang S, et al: MiR-126 suppresses colon cancer cell proliferation and invasion via inhibiting RhoA/ROCK signaling pathway. Mol Cell Biochem 380: 107-119, 2013. 